\section{Integrability in Classical and Quantum Field Theory and the Bukhvostov-Lipatov Model ${ }^{1}$}

\author{
Bogomil Gerganov \\ Nevman Loboratory of Nuclear Studices \\ Cornell University \\ Abatract. A brief overview of the notions of classical and quantum integrability in \\ $1+1$ spece-time dimensions is given with an emphasis on the existence of infinite \\ number of conserved quantities in $(1+1) D$ Geld theories. Same methods for building \\ such conserved quantities are outlined and are then spplied to study the integrability \\ Thirring model. The integrability properties of both the cleseical/quantum and the \\ boeonic/termionic versions of the model are discussed. It is shown that the classica) \\ fermionic model posesses a conserved current of Lorent $z$ spin 3 . It is then establiahed \\ that the conservation law is spoiled at the quantum level - A fact that indicates that \\ the quantum Bukhvostov-Lipatov model is not integrsble.
}

\section{INTEGRABILITY IN 1 + 1 SPACE-TIME \\ DIMENSIONS}

\section{A Classical Integrability}

Let the time evolution of a physical system be described by some (generally nonlinear) partial differential equations. The system is said to be classically integrable if there exist exact integrals to its classical equations of evolution.

The pioneering work in the field of classically integrable non-linear systems was a 1967 paper by Gardner Greene, Kruskal, and Miura [1]. The authors were a 1967 peper by Gardner, Gre Gre variables that makes the Korteweg-de

\footnotetext{
1) Talk given at MRST XX, McGill University, Montrésl, May 13-15, 1998
}

developing a general procedure [2] for associating to a system of non-linear PDEs a linear operator, whose eigenvalues are integrals of the non-linear system.

In the following decades exact solutions to a number of non-linear clasaical sys tems have been found and the importance of the field has grown for several reasons:

The evolution of some very important physical systems is governed by non-linear equations that admit exact solutions. Their range of applicability includes fuic dynamics, non-linear optics, and condensed matter theory.

Most known classically integrable systems exhibit the presence of "particle-like" solutions to the non-linear equations of motion - solitary waves or solitans. Their properties present interest to many areas of physics, both applicable, such as optic fiber design, and purely theoretical, such as string theory.

The solution of enme integrable oystems can be ued as $0^{\text {th }}$-order approximations in perturbation theory to study non-integrable systems. Such refined perturbative in perturbation theory to study non-integrable systems. Such reined perturbet

Some examples of integrable non-linear partial differential equations (PDEs) are:

- Korteweg-de Vries equation (KdV): $\phi_{t}+6 \phi \phi_{x}+\phi_{x+x}=0,[1,2]$

- Non-linear Schödinger equation: $i \phi_{t}=-\phi_{x x}+|\phi|^{2} \phi,\{1,2]$.

- sine-Gordon equation (sG): $\phi_{t t}-\phi_{x x}=\sin \phi,[3]$.

A comprehensive introduction to the subject of classical integrability and the theory of sobitons can be found in $[4]$.

B Quantum Integrability in $1+1$ Space-Time Dimensions

Because of the specific (strongly restrictive) features of particle kinematics in $(1+1)$-dimensional space-time, there exist scattering theories which allow precise computation of all elements of the exact particle $S$-matrix. This is possible because the following 2 (related) properties, esch of which can be taken as a definition of quantum integrability in $(1+1) D$

Definition A. A QFT in 2 space-time dimensions is integrable if there exists an infinite set of independent, mutually commuting integrals of motion

$$
\left[Q_{r}, H\right]=0, \quad\left[Q_{r}, Q_{r}\right]=0, \quad r=1,2,3, \ldots
$$

Definition B. A QFT in 2 space-time dimensions is integrable if the $N$-paticle $S$-matrix can be factorized into a product of $\frac{N(N-1)}{2}$ 2-particle $S$-matrices, as if the process of $N$-particle scattering were reduced to a sequence of pair collisions; thus, allowing all the matrix elements of $S$ to be determined exsctly. 
It can be shown that Property B follows from Property A. Indeed, in $1+1$ dimensions the ouly symmetry transformations on the space-time are the translation and the Lorentz boosts That's why, in the limits $t \rightarrow \pm \infty$, any conserved tensorial quantity of rank $r$ reduces to a polynomial in the asymptotic particle 2-moinents. In a diagonal basis this polynomial could be written a

$$
\hat{Q}_{r}=\sum_{a \in I N} \hat{p}_{a}^{r}=\sum_{\omega \in O U T} \hat{p}_{b}^{r}
$$

The last result is important because it has 2 consequences:

1. No particle production (the $S$-matrix is elastic)

Let $\left|p_{1}, p_{2}, \ldots, p_{a}, \ldots, p_{N_{a}}\right\rangle_{I N}$ and $\left|p_{1}^{\prime}, p_{2}^{\prime}, \ldots, p_{b}^{\prime}, \ldots, p_{N_{b}}^{\prime}\right\rangle_{O U T}$ be asymptotic operator $Q_{r}$, we obtain, according to (1.2), that

$$
\sum_{a=1}^{N_{a}} p_{a}^{r}=\sum_{b=1}^{N_{b}} p_{b}^{r}
$$

Since there are infinite number of such conserved charges in the theory, the particle 2-momenta sholud satisfy infinite number of conservation laws like (1.3) with $r=1,2,3, \ldots$ This is only possible if the set of the ingoing 2 monenta is equal to the set of outgoing 2-momenta:

$\left\{p_{1}, p_{2}, \ldots, p_{a}, \ldots, p_{N_{a}}\right\}_{I N}=\left\{p_{1}^{\prime}, p_{3}^{\prime}, \ldots, p_{b}^{\prime}, \ldots, p_{N_{b}}^{\prime}\right\}_{\text {OUT }}, \quad N_{a}=N_{b}=N$

This implies, in particular, that the total particle number and the number of particles of given mass, $m^{2}=p_{\mu} p^{4}$, is conserved. Therefore,

$$
S_{f_{2}}=\operatorname{our}\left\langle p_{1}^{\prime}, p_{2}^{\prime}, \ldots, p_{b}^{\prime}, \ldots, p_{N}^{\prime} \mid p_{1}, p_{2}, \ldots, p_{a}, \ldots, p_{N}\right\rangle_{I N} \text { is elastic. }
$$

2. Multiparticle collisions reduce to a sequence of pair collisions (the $S$-matrix is factorizable).

Since there exists an infinite set of integrals of motion in our theory, it is natural to assume that there is a particular symmetry transformation that reduces at $t \rightarrow \pm \infty$ to independent translations of the individual particles. By performing such translations on can get arbitrarily large space-time separations of all the regions where pair collisions occur. Therefore, the pair collisioins can be treated as independent. The existence of infinite number of symmetrie further implies that the time order of the collisions is irrelevant and, therefore, different permutations of the 2-particle $S$-matrices in the factorized product will give rise to the same nultiparticle $S$-matrix (Yang-Baxter equations)

The above 2 properties, together with the Yang-Baxter equations and the requirements for unitarity, analyticity, and crossing symmetry, for many $(1+1) D$ QFTs provide enough constraints to compute the $S$-matrix exactly.

extensive review of the properties of the $S$-matrix in $(1+1) D$ can found in [5]

\section{METHODS FOR BUILDING INTEGRALS OF MOTION OF HIGHER TENSORIAL RANK}

\section{A Classical Integrals of Motion}

In a remarkable paper [2] Lax formulated a powerful technique for integrating exactly systems of non-linear evolution equations. Let

$$
u_{t}=K(u)
$$

be some non-linear PDE and let $u(x, t)$ be a solution to (II.1). Lax showed that if we can associate some selfadjoint linear differential operator $L_{u}$ with the function $u$, such that $\hat{L}_{u}$ is unitary invariant under the evolution of $u$ according to (II.1), then the eigenvalues of $\hat{L}_{u}$ are integrals of motion of (II.1)

If we find the operator $\hat{L}_{v}$ for a certain PDE, it generates an infinite series of integrable PDEs. The higher order PDEs in the series can be used to build integrals of motion of higher rank and, thus, to construct and infinite series of conservatio laws of the classical system. Using this and a variety of other methods, infinite set of conserved quantities of increasing spin have been constructed for the classical MT, Korteweg-de Vries (KdV), and sG models $[1-3,6,7]$.

\section{B Quantum Integrals of Motion}

A powerful tool for building conserved quantities for $2 D$ quantum models is the technique of perturbed conformal field theory [8]. By treating a $2 D$ QFT as a perturbed CFT, one can make use of conformal invariance to find an infinite number of conserved quantities in the conformal theory. It is then possible to study which (if any) of these conservation laws survive the pert urbation. Zamolodchikov's paper [8] provides an easy way of computing the conserved current densities explicitly.

In CFT any conserved density $T_{t+1}$ is a holomorphic function and $\partial_{\varepsilon} T_{t+1}=0$ In the perturbed QFT that is no longer true and we can compute $\partial_{\varepsilon} T_{t+1}$, using Zamolodchikov's formula [8, eq.(3.14)]:

$$
\partial_{\bar{z}} T_{t+1}=\lambda \oint_{z} \frac{d \zeta}{2 \pi i}: \Phi(\zeta, \bar{z}):: T_{s+1}(z)
$$

If the RHS of (II.2) can be expressed as a total $\partial_{z}$-derivative of some local operator $\Theta_{,-1}$, there exists a spin $s$ conservation law surviving in the perturbed QFT:

$$
\partial_{z} T_{*+1}=\partial_{z} \Theta_{,-1}, \quad \partial_{z} \bar{T}_{*+1}=\partial_{\bar{z}} \bar{\Theta}_{--1},
$$

$T_{0+1}$ and $\Theta_{,-1}$ being the quantum conserved densities. 


\section{THE BUKHVOSTOV-LIPATOV MODEL}

The bosonic Bukhvostov-Lipatov model (BL) is a 2-field generalization of the sine-Gordon model $(\mathrm{sG})^{2}$. It was first introduced by Bukhvostov and Lipatov [10] in a study of the $O(3)$-nonlineur $\sigma$-model and has drawn recent attention in works by Fateev [11] and Lesage, Saleur, Simonetti [12]. The model is defined by the action

$$
S=\frac{1}{4 \pi} \int d t d x\left[\frac{1}{2}\left(\partial_{\mu} \phi_{1}\right)^{2}+\frac{1}{2}\left(\partial_{\mu} \phi_{2}\right)^{2}+\lambda: \cos \hat{\beta}_{1} \phi_{1}:: \cos \hat{\beta}_{2} \phi_{2}:\right],
$$

where $\hat{\beta}_{i} \cong \hat{\beta}_{i}, i=1,2$. Using the and Coleman's bosonization prescription \{13\}, Bukhvustov and Lipatov mapped ${ }^{3}$ the quantum bosonic theory (III.1) to a dual theory of two Dirac fermions

$$
\begin{gathered}
S_{\text {full }}=\frac{1}{4 \pi} \int d t d x\left\{\frac{i}{2} \Psi^{D} \gamma^{\mu} \partial_{\mu} \Psi-\frac{i}{2} \partial_{\mu} \Psi^{D} \gamma^{\mu} \Psi+\frac{i}{2} X^{\mathcal{D}} \gamma^{\mu} \partial_{\mu} X-\frac{i}{2} \partial_{\mu} X^{D} \gamma^{\mu} X\right. \\
\left.-m\left(\Psi^{\mathcal{D}} \Psi+X^{\mathcal{D}} X\right)-g\left(\Psi^{\mathcal{D}} \gamma^{\mu} \Psi\right)\left(X^{\mathcal{D}} \gamma^{\mu} X\right)-g^{\prime}\left[\left(\Psi^{\mathcal{D}} \gamma^{\mu} \Psi\right)^{2}+\left(X^{\mathcal{D}} \gamma_{\mu} X\right)^{2}\right]\right\} .
\end{gathered}
$$

where

$$
g=\pi^{2}\left(\frac{1}{\hat{\beta}_{1}^{2}}-\frac{1}{\hat{\beta}_{2}^{2}}\right), \quad g^{\prime}=\frac{\pi^{2}}{2}\left(\frac{1}{\hat{\beta}_{1}^{2}}+\frac{1}{\hat{\beta}_{2}^{2}}-4\right) .
$$

The above model is ubviously a 2 -field generalization of the massive Thirring model (NTM). It involves 2 types of 4-fermion interactions: the interaction of the MT model with coupling $g^{\prime}$ and a new interaction term with coupling $g$.

In [10] Bukhvostov and Lipatov studied the integrability of the model (III.2) using Bethe ansatz [14]. They constructed the $S$-matrix for the pseudoparticles and showed that it satisfies Yang-Baxter equations in 2 cases
1. $g=0$,
$g^{\prime}:$ unrestricted
(III.4)
2. $g^{\prime}=0$,
$g$ : unrestricted
(III.5)

In Case 1 (HII.2) reduces to two decoupled copies of the MT model, known to be integrable both classically and quantum mechanically $[6,15]$. In Case 2 one obtains a new model, to which we will refer as "the fermionic BL model":

$$
S_{B L}=\left.S_{\text {full }}\right|_{g^{\prime}=0}
$$

\footnotetext{
2) Generalizations of the sine-Gordon model have been studied as early as 1981 [9].

3i After a rotation of the boson fields: $\phi_{1}=\frac{1}{2 \xi_{1}}\left(v_{1}+v_{2}\right)$ and $\phi_{2}=\frac{1}{2 \phi_{2}}\left(v_{1}-v_{2}\right)$.

1) Here $\psi^{D}$ dewotes the Dirac conjugate of $\psi, \psi^{D} \equiv \psi^{\dagger} \gamma^{0}$. We use this unconventional notation

rather than the usual " $\bar{W}$ " to avoid confusion with the antichiral spinor component, $\bar{\psi}$.
}

Based on their result for the pseudoparticle $S$-matrix, Buklivestov and Lipatov conjectured that the quantum field theory (III 6) is integrable. The actual physical cont kent knowledge, compating the physical $S$-mall remaius an open problem.

The

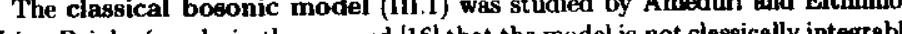
Using Painleve asalysis, they proved [16] that the model is not clasically integrable. In the following sections we discuss in sone details the in fermionic model (III.6) and the quantum integrabillty of the models (III.1) and (III.2), dual to each other in the quantum case. We focus, in particular, constructing conserved quantities of higher tensorial rank in these theories.

\section{A Classical Fermionic Model}

In Eucledian spece-time, parametrized by the light cone coordinates $z=$ $\frac{1}{2}(\tau+i x), \bar{z}=\frac{1}{2}(\tau-i x)$, and in terms of spinor components ${ }^{5}$, the action (III.6) becones

$$
\begin{aligned}
& S_{E}=\frac{1}{4 \pi} \int d \tau d x\left[\frac{1}{2}\left(\psi_{+} \partial_{\xi} \psi_{-}+\psi_{-} \partial_{\xi} \psi_{+}+\bar{\psi}_{+} \partial_{x} \bar{\psi}_{-}+\bar{\psi}_{+} \partial_{z} \bar{\psi}_{-}\right)+\right. \\
& \left.-i m\left(\bar{\psi}_{+} \psi_{-}+\bar{\psi}_{-} \psi_{+}\right)-g\left(\psi_{+} \psi_{-} \bar{\chi}_{+} \bar{\chi}_{-}+\bar{\psi}_{+} \bar{\psi}_{-} \chi_{+} \chi_{-}\right)+(\psi \rightarrow \chi)\right],
\end{aligned}
$$

which gives rise to the classical equations of motion

$\partial_{z} \psi_{ \pm}(z, \bar{z})=-i m \bar{\psi}_{ \pm} \pm 2 g \psi_{ \pm} \bar{\chi}_{+} \bar{X}_{-}, \quad \partial_{s} \bar{\psi}_{ \pm}(z, \bar{z})=i m \psi_{ \pm} \pm 2 g \bar{\psi}_{ \pm} \chi_{+} \chi$

and the equations obtained with the substitutiuon $\psi \rightarrow \chi$.

Because of the space and time translational invariance, the theory possesses a trivial conserved charge of spin 1 - the energy-momentum tensor. Finding conserved quantitites of higher Lorentz spin is equivalent to finding non-trivial integrals of motion and is considered to be strong indication for the classical integrability of the theory. We have been able to show [17] that the fermionic BL model (III.7) has a classically conserved charge of spin 3 in the bulk:

$$
Q_{3}=\int_{-\infty}^{+\infty} d x\left(T_{4}-\Theta_{2}\right) \text { and } \bar{Q}_{3}=\int_{-\infty}^{+\infty} d x\left(\bar{T}_{4}-\bar{\Theta}_{2}\right)
$$

\section{We use the following convention}

$$
\Psi \rightarrow\left(\begin{array}{l}
\psi_{-} \\
\bar{\psi}_{-}
\end{array}\right), \psi^{\top} \rightarrow\left(\psi_{+} \bar{\psi}_{+}\right) ; \psi^{\mathcal{D}} \equiv \boldsymbol{\psi}^{\mathrm{\gamma}} \gamma^{0} ; \gamma=\left[\begin{array}{ll}
0 & 1 \\
1 & 0
\end{array}\right], \quad \gamma_{1}=\left[\begin{array}{cc}
0 & 1 \\
-1 & 0
\end{array}\right]
$$

and, similarty, for $X$. The components of the neetric tensor are $\left[g_{\mu \nu}\right]=\left[g^{\mu \nu}\right]=\operatorname{diag}[1,-1]$. 
where the densities $T_{4}$ and $\Theta_{2}$ are given by:

$$
\begin{aligned}
T_{1} & =\psi_{+} \partial_{z}^{3} \psi_{-}+6 g \partial_{z} \psi_{+} \partial_{z} \psi_{-} \chi_{+} \chi_{-}+(+\leftrightarrow-)+(\psi \leftrightarrow \chi), \\
\Theta_{2} & =i m \bar{\psi}_{+} \partial_{z}^{2} \psi_{-}+2 m g \bar{\psi}_{+} \partial_{z} \psi_{-} \chi_{+} \chi_{-}+4 m g \bar{\psi}_{+} \psi_{-} \chi_{+} \partial_{x} \chi_{-}+ \\
& +4 m g \bar{\psi}_{+} \psi_{-} \partial_{z} \chi_{+} \chi_{-}-4 i m^{2} g \psi_{+} \psi_{-} \chi_{+} \chi_{-}+(+\leftrightarrow-)+(\psi \mapsto \chi)
\end{aligned}
$$

(similarly, for $\bar{T}_{1}$ and $\bar{\Theta}_{2}$ ) and satisfy the continuity equation (II.3) with $s=3$. The spin 3 charge (III.9) is conserved also in the theory on the half-line for specific types of boundary actions $[17\}$. We intend to generalize our result to conserved quantities of arbitrary spin by using methods similar to the ones described in Refs. $[1-3,6,7]$. We have also found that the more general 2-fermion model (III.2) does not possess a classically conserved spin 3 current for arbitrary values of the couplings $g$ and $g^{\prime}$. Classical conservations laws of spin 3 exist only in the special cases $g=0$ $\left(2 \times\right.$ MTM) and $g^{\prime}=0$ (BL model), i. e. the model (III.2) is classically integrable precisely in the cases (III.4) and (III.5), suggested by Bukhvostov and Lipatov.

\section{B Quantum Integrability}

In this section we will study the modifications to the classical conservation law due to quantum corsections, using perturbed conformal field theory ${ }^{6}$. Bosonfermion duality in 2 space-time dimensions allows us to write our expression for the classically conserved fermionic current (III.10) in bosonic form and then to study its quant um conservation in the model (III.1), treating the term $\lambda: \cos \hat{\beta}_{1} \phi_{1} \cos \hat{\beta}_{2} \phi_{2}$ as a single relevant perturbation to the conformul theory of massess free bosons. Using the substitutions $\psi_{ \pm}=e^{ \pm i \theta_{1}}, \chi_{ \pm}=e^{ \pm i \theta_{2}}$. and the vertex operator product Using the sut expansions from $C F T \mid 18\}$, we can map each term of $T_{4}$ to its dual operator in the bosonic theory. of the classical $T_{1}$, we prefer to leave them as arbitrary hactions of $g$ and fix them relations in Font relations in Foots

$$
T_{4}=\sum_{m+n=2} a_{m n}:\left(\partial_{2}^{2} \phi_{1}\right)^{m}\left(\partial_{z}^{2} \phi_{2}\right)^{n}:+\sum_{k+1=4} b_{k t}:\left(\partial_{2} \phi_{1}\right)^{k}\left(\partial_{2} \phi_{2}\right)^{l}:
$$$$
+c_{1}:\left(\partial_{z} \phi_{1}\right)^{2} \partial_{z}^{2} \phi_{2}:+c_{2}: \partial_{z}^{2} \phi_{1}\left(\partial_{z} \phi_{2}\right)^{2}:
$$

This is, in fact, the most general ansatz for $T_{4}$ for the double cosine model includes, up to addition of total $\partial_{z}$-derivatives, all operators of mass dimension 4 with arbitrary coefficents, functions of $\left(\hat{\beta}_{1}, \hat{\beta}_{2}\right)$ or via (III) 3 ) of $g$. As it turas out whe arpitrat, gives enough information to compute the exact form of these functions.

6) See Section II. B and Ref. [8].
In starting this calculation, our goal was to find all the conditions on the couplings $\hat{\beta}_{1}$ and $\hat{\beta}_{2}$ for which the spin 3 charge is conserved. We expected that the 'BL manifold' (III.5) would be one of the integrable cases and then, by fermionizing back, we would be able to obtain an exact quantum expression for the fermionic $T_{1}$. At the end, we found that the spin 3 current is conserved only in 3 cases:

$$
\begin{aligned}
& \hat{\beta}_{1}^{2}-\hat{\beta}_{2}^{2}=0 \longrightarrow g=0 \\
& \hat{\beta}_{1}^{2}+\hat{\beta}_{2}^{2}=1 \longrightarrow\left(\frac{2 g^{\prime}}{\pi^{2}}+2\right)^{2}-\left(\frac{g}{\pi^{2}}\right)^{2}=4 \\
& \hat{\beta}_{1}^{2}+\hat{\beta}_{2}^{2}=2 \longrightarrow\left(\frac{2 g^{\prime}}{\pi^{2}}+3\right)^{2}-\left(\frac{g}{\pi^{2}}\right)^{2}=1
\end{aligned}
$$

The first manifold is trivial: when $\hat{\beta}_{1}^{2}=\hat{\beta}_{2}^{2}$ the double cosine model decouples into 2 sine-Gordon modeks, integrable both classically and quantum mechanically. The second two ${ }^{7}$ quantum integrable ${ }^{8}$ manifolds have been previously identified by Fateev [11] and by Lesage, Saleur, and Simonetti [12]. On the BL manifold (III.5) the charge (III.9) is not quantum conserved, except in the trivial case when the manifolds (III.13), (III.5), and (III.14) intersect each other (free fermion point).

The above result becomes even more clear when we look at the fermionic parameter space. As we showed at the end of Section III.B, the general model has a classically conserved charge of spin 3 only if either $g=0$ or $g^{\prime}=0$. Therefore, the classical integrable manifolds are simply the axes of the $\left(g, g^{\prime}\right)$-plane

We see that the manifold $g=0$ is present both in the classical and in the quantum case. This merely reflects the fact that massive Thirring model is integrable both classically and quantum mechanically. In other words, if we start with the classical MT model, turning on quantum corrections will not take the classical conservation law away from the vertical axis. The coefficients of $T$, will be modified but they will still remain functions of $g^{\prime}$ only and will be independent of $g$.

In contrast, if we start with the classical fermionic BL model, with $T_{4}$ dependin on $g$ only (III.10), quantum corrections will not only modify the coefficients of the conserved current but will also introduce new terms, involving $g^{\prime}$-dependence Looking at Figure 1, we see that the $g$-axis, on which the model is integrable classicaily, is 'deformed' into the hyperbola (III.14) in the quantum case. The only point on the classical manifold where integrability is preserved by quantum mechanics is the origin, which, of course, is simply the the free point $\left(g=g^{\prime}=0\right.$ ) of the fermionic theory.

\footnotetext{
7) We concentrate bere on the first non-trivial manifold, (III.14), since this is the one with physical siguificance (tunneling in quasnt unu wires) [12]

but is quantum integrable on certain sub

manifolds of the parameter spece has been previously discussed by Shanlar
} 


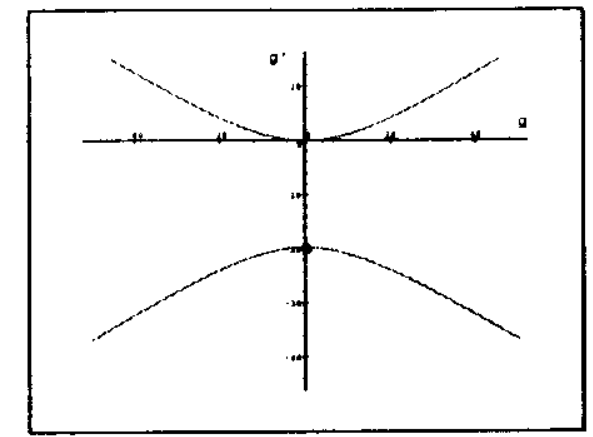

FIGURE 1. Fermionic parameter space.

\section{CONCLUSION}

We have shown that the fermionic Bukhvostov-Lipatov model, given by the action (IIl.6), admits a nontrivial classical integral of motion of spin 3. This conservatou law holds quantum mechanically only at the free fermion point $g=0$ and is spoiled by quantum corrections for generic values of the coupling $g$. The more general fermionic model (III.2) admits a quant um conservation law of spin 3 for the specific relation (III.14) between the couplings $g$ and $g^{\prime}$. The study of the spin 3 conservation laws, therefore, suggests that the integrable manifold $\left(g\right.$ : free, $g^{\prime}=0$ ) proposed by Bukhvostov and Lipatov does not survive in the quantum field theory.

\section{ACKNOWLEDGEMENTS}

I would like to thank my colliaborators Costas Efthimiou and Marco Ameduri for completing this work. I would also like to thank André LeClair for many useful discussions and insights on the subject and to Frédéric Lesage for a discussion on the double-cosine mode

\section{REFERENCES}

1. Gardner, C. S., Greene, J. M., Kruskal, M. D., and Miura, R. M., Phys. Rev. Let 18, $1095(1967)$

2. Lax, P. D., Comm. Pure Appl. Math. 21, 467 (1968).

3. Takitadzhyan, L. A., and Faddeev, L. D., Theor. Math. Phys. 21, 1046 (1974)

Faddeev, L. D., Takhtajan, L. A., Kamulontan Methods in the Theory of Solitons, Berlin/Heidelber: Springer-Verlag, 1987, and the references therein.
Zumolodchikov, A. B. in Soviet Scientific Reviews, Sec. A, Khalatnikow, I. M., ed. New York: Harwood Academic Publishers, 1980, vol. 2, pp. 1-40.

(1976); Fiune, R. Mitter P K and Papanich N Phys. Lett B64 289 (1976).

7 Ferrars, S. Girardello, L., and Sciuto, S., Phys. Lett. B76, 303 (1978); Giraradello, L., and Sciuto, S., Phys. Lett. B77, 267 (1978).

Zanolodchikov, A. B., Adv. Studies in Pure Math. 19, 641 (1989).

8. Zannoloddhiko, A. B., Adv. Sludses in Pure $M$

0. Bukhvostov, A. P., and Lipatov, L. N., Nucl. Phys. B180, 116 (1983)

1. Fateev, Y. A. Nucl. Phys. B473, 509 (1996).

12. F. Lesage, F., Saleur, H., and Simonetti, P., Tunneling in Quantum Wires I: Exact Solution of the Spin Isotropic Case, cond-nat/9703220; Tinneling in Quantum Wire II: A New Line of IR Fixed Points, cond-mat/9712019.

13. Coleman, S., Phys. Rev. D11, 2088 (1975).

14. Korepin, V. E., Bogoliubov, N. M., and Izergin, A. G., Quantum Inverse Sonttering Method and Comelation Functions, Cambridge: Cambridge University Press, 19

15. Kulish, P. P., and Nisimow, E. R., Pis'ma 2h. Eksp. Teor. Fiz. 24, 247 (1976).

16. Ameduri, M., Ethimiou, C., J. Nonl. Malh. Phys. S, 132 (1998).

17. Ameduri, M., Efthimiou, C., and Gerganoy, B. The Bukhnstov-Lipatov Model

18. Di Francesco, P., Mathieu, P., and Sénéchal, D., Conformal Field Theory, New York Spinger, 1997 